\section{A maturing European Research Council}

\section{The European Research Council aims to support frontier research by individual research groups. As the organization enters its fourth year, we hope that it will be better able to implement these scientific goals.}

The European Union has long needed a pan-European organization to fund cutting-edge research performed by individual investigators. National programmes that support scientific research must ensure that their funds are widely distributed, which could result in a larger number of awardees receiving smaller grants. By contrast, an EU-wide funding agency with large sums of money at its disposal can disperse significantly larger grants to a smaller number of grantees, and do so without the risk of depriving a majority of the research community. Such an agency can also ensure that challenging new projects with potential are funded appropriately. National programmes are driven by state-specific interests, whereas an EU-wide organization should identify and prioritize research interests that develop the European science community as a whole. Although previous EU funding programmes, such as the Networks of Excellence, have prioritized large research projects that involve collaborations across EU countries, supporting excellence in basic research conducted by individual research groups is also crucial. The European Research Council (ERC), established in 2007, promised to redress these deficiencies in the EU science funding landscape.

With a budget of $€ 7.5$ billion extending from 2007 to 2013, and a stated mission to fund the best and most innovative research in science and engineering, the ERC was highly anticipated to provide a significant boost to European research. However, the ERC has an annual budget of about $€ 1$ billion, which is much smaller than that of the NSF ( $\$ 7$ billion) or the NIH ( $\$ 28$ billion), and covers a population (EU and associated states) almost twice of that of the US (610 million versus 308 million). The ERC, therefore, cannot supplant national funding agencies, which must continue to provide the bulk of research funding. Nevertheless, the ERC was a welcome addition, complementing existing national programmes, and offering a glimmer of hope for scientists in countries suffering from budget cuts in basic research funding.

At present, the ERC operates two grant programmes, one for starting scientists and the other for senior researchers. The size of the grants awarded to senior researchers is considerable. For example, in 2010, 89 researchers received Advanced Investigator Grants in Life Sciences of up to $€ 3.5$ million for 5 years. Carl-Henrik Heldin, a member of the ERC scientific council, points out that the main criterion for these awards is the quality of research over the past ten years. This ensures that only active scientists who continue to make strong contributions are rewarded. "Having received the Nobel Prize 15 years ago should not make a difference," says Heldin.

Perhaps more significantly, the ERC also provides substantial support for junior faculty members. In some European countries, start-up funding is either absent or insufficient. The ERC's lavish Starting Independent Researcher Grants of around $€ 1.5$ million extending over five years is generous by any measure. Even prestigious national programmes, such as the Wellcome Trust in the UK, distribute smaller annual grants to new PIs. However, the size of the starting awards limits the number of successful applicants: only 80 grants were given for Life Sciences in 2009. A welcome spin-off effect is that national funding programmes in several countries have decided to fund 'runners-up', who did not meet the bar for ERC grants, without further applications and review. Thus, the size of the ERC grants should not only ensure that starting scientists can establish independent research programmes without having to struggle for additional sources of funding, but it should also enable ambitious, large-scale projects that might require significant investment in new technology and reagents.

Another current obstacle in research funding is the tendency not to compare like with like: new PIs are not differentiated from those with more experience but still 'young', when competing for career development grants. The ERC has taken an important step towards addressing this problem. The budget for the Starting Independent Researcher Grants is now split so that 'real starters', with 2-6 years of postdoctoral experience, are not competing directly with 'consolidators' with 6-10 years of postdoctoral experience.

Although the ERC has been met with much enthusiasm from the European research community, its early years have not passed without problems. The scientific council seems to have done a remarkable job in launching the funding project and attracting top scientists within and outside Europe as reviewers and panel members. The selection of candidates has also been generally acclaimed. However, the dayto-day administration, which has been done by administrators within the Commission, is cumbersome. Grantees, panel members and grant reviewers have complained about excessive bureaucracy. Indeed, Professor Fotis Kafatos, who has recently stepped down as Chair of the Scientific Council and has been succeeded by the former Vice-Chair, Professor Helga Nowotny, also noted that this was a hurdle to be overcome. Integrating the scientific goals of the ERC with the grant management would be improved if a scientist headed the grant administration programme. This was indeed recommended in 2009 by a heavyweight independent review panel. In response to the panel's numerous recommendations and bluntly worded critique, ERC management has been lifted out of the European Commission and the ERC will be appointing a senior scientist with administrative experience as its director who, we hope, will be able to implement the necessary reforms. The long-term success of the ERC depends on the ability of its new administrators and leaders to work without political interference to further the interests of a European science agenda. 